\title{
Note on $\boldsymbol{H}$-separable extensions
}

Dedicated to Professor Kiiti Morita on his 60th birthday

By Taichi Nakamoto and Kozo Sugano

It is the purpose of this note to give a (self-contained) computational proof to the principal theorem (1.3) of [7] and a theorem concerning ringendomorphisms of an $H$-separable extension. Our tool employed in this note is an $H$-system of an $H$-separable extension, which was introduced in [4].

Recently, we found that the proof of [6, Proposition 1] contained an error and the same was repeated in the proof of main part of [7, (1.3)]. So, the present note comprehends the correction to the previous papers [6] and [7].

Throughout, $A / B$ will represent a ring extension with common identity $1, V$ the centralizer $V_{A}(B)$ of $B$ in $A$, and $C$ the center of $A$.

The next will be useful occasionally in the subsequent study.

(1) Let $B^{\prime} \subset B^{\prime \prime}$ be intermediate rings of $A / B$. Let $V^{\prime}=V_{A}\left(B^{\prime}\right)$, and $V^{\prime \prime}=V_{A}\left(B^{\prime \prime}\right)$. If ${ }_{B^{\prime}} B^{\prime} \otimes_{B} B^{\prime \prime}{ }_{B^{\prime \prime}} \rightarrow{ }_{B^{\prime}} B^{\prime \prime}{ }_{B^{\prime \prime}}\left(b^{\prime} \otimes b^{\prime \prime} \mapsto b^{\prime} b^{\prime \prime}\right)$ splits then ${ }_{V^{\prime}} V_{V^{\prime \prime}}^{\prime \prime}$ $<\oplus_{\nabla^{\prime}} V_{\nabla^{\prime \prime}}$.

Proof. There exists an element $\sum_{k} b_{k}^{\prime} \otimes b_{k}^{\prime \prime} \in\left(B^{\prime} \otimes_{B} B^{\prime \prime}\right)^{B^{\prime}}$ such that $\sum_{k} b_{k}^{\prime} b_{k}^{\prime \prime}=1$. Then, the map $q: V \rightarrow V^{\prime}$ defined by $v \mapsto \sum_{k} b_{k}^{\prime} v b_{k}^{\prime \prime}$ is a $V^{\prime}-V^{\prime \prime}$-homomorphism and induces the identity map on $V^{\prime}$, which means ${ }_{V^{\prime}} V^{\prime}{ }_{V^{\prime \prime}}<\oplus_{V^{\prime}} V_{V^{\prime \prime}}$.

$A / B$ is called an $H$-separable extension if $A \otimes_{B} A$ is $A$ - $A$-isomorphic to an $A$ - $A$-direct summand of a finite direct sum of copies of $A$. To be easily seen, $A / B$ is $H$-separable if and only if there exist some $v_{i} \in V$ $(i=1, \cdots, m)$ and $\sum_{j} x_{i j} \otimes y_{i j} \in\left(A \otimes_{B} A\right)^{A}$ such that $\sum_{i, j} x_{i j} \otimes y_{i j} v_{i}=1 \otimes 1$. Following [4], such a system $\left\{v_{i} ; \sum_{j} x_{i j} \otimes y_{i j}\right\}_{i}$ will be called an $H$-system for $A / B$.

In what follows, we assume always $A / B$ is an $H$-separable extension with an $H$-system $\left\{v_{i} ; \sum_{j} x_{i j} \otimes y_{i j}\right\}_{i}$. Then the map $\eta: A \otimes_{B} A \rightarrow$ $\operatorname{Hom}_{C}(V, A)\left(a_{1} \otimes a_{2} \mapsto\left(v \mapsto a_{1} v a_{2}\right)\right)$ is an $A$-A-isomorphism, whose inverse is given by $h \mapsto \sum_{i, j} x_{i j} \otimes y_{i j} h\left(v_{i}\right)$ (cf. also $\left(2.1^{\prime}\right)$ ).

(2) If $\sigma$ is an arbitrary ring-endomorphism of $A$ which leaves every element of $B$ invariant, $g \in \operatorname{Hom}\left(A_{B}, A_{B}\right)$ and $h \in \operatorname{Hom}\left({ }_{B} A,{ }_{B} A\right)$, then 


$$
\sum_{i, j} g\left(x_{i j}\right) v \sigma\left(y_{i j}\right) \sigma(a) \sigma\left(v_{i}\right)=g(a) v
$$

and

$$
\sum_{i, j} \sigma\left(v_{i}\right) \sigma(a) \sigma\left(x_{i j}\right) v h\left(y_{i j}\right)=v h(a) \quad(a \in A, v \in V) .
$$

PROOF. $\quad \sum_{i, j} x_{i j} \otimes y_{i j} a v_{i}=a \otimes 1$ implies $\sum_{i, j} g\left(x_{i j}\right) \otimes \sigma\left(y_{i j} a v_{i}\right)=g(a) \otimes 1$. Applying $\eta$, we obtain (2.1).

The above formulae are specialized in various ways:

$$
\begin{gathered}
\sum_{i, j} \sigma\left(x_{i j}\right) v \sigma\left(y_{i j}\right) \sigma\left(v_{i}\right)=\sum_{i, j} \sigma\left(v_{i}\right) \sigma\left(x_{i j}\right) v \sigma\left(y_{i j}\right)=v . \\
\sum_{i, j} g\left(x_{i j}\right) v y_{i j} a v_{i}=g(a) v . \\
\sum_{i, j} v_{i} a x_{i j} v h\left(y_{i j}\right)=v h(a) .
\end{gathered}
$$

In particular, we have $\sum_{i, j} v_{i} x_{i j} v y_{i j}=v$, which means:

(3) $V_{c}$ is f.g. (finitely generated) projective ([3, p. 112]).

(4) $A / B$ is a separable extension ([3, Theorem 2.2]).

Proof. Since $V_{c}$ is f.g. projective by (3), there exists a $C$-epimorphism $q: V \rightarrow C$ which induces the identity map on $C$. Obviously, $\sum_{i, j} x_{i j} \otimes y_{i j} q\left(v_{i}\right)$ is in $\left(A \otimes_{B} A\right)^{A}$ and $\sum_{i, j} x_{i j} y_{i j} q\left(v_{i}\right)=q\left(\sum_{i, j} x_{i j} y_{i j} v_{i}\right)=1$, which means that $A / B$ is separable.

Next, by a brief computation with $\left(2.1^{\prime}\right)$ and $\left(2.2^{\prime}\right)$, we see that the map $\xi: V \otimes_{C} V \rightarrow \operatorname{Hom}\left({ }_{B} A_{B},{ }_{B} A_{B}\right)\left(u_{1} \otimes u_{2} \mapsto\left(a \mapsto u_{1} a u_{2}\right)\right)$ is a $V$ - $V$-isomorphism, whose inverse is given by $h \mapsto \sum_{i} \sum_{j} h\left(x_{i j}\right) y_{i j} \otimes v_{i}=\sum_{i} v_{i} \otimes \sum_{j} x_{i j} h\left(y_{i j}\right)$.

(5) If ${ }_{B} B<\oplus_{B} A$ or $B_{B}<\oplus A_{B}$ then $V_{A}(V)=B$ ([5, Proposition 1.2]).

Proof. Let $p: A \rightarrow B$ be a left $B$-epimorphism which induces the identity map on $B$. Then, for $a \in V_{A}(V)$ we have $p(a)=\sum_{i, j} v_{i} a x_{i j} p\left(y_{i j}\right)$ $=a \sum_{i, j} v_{i} x_{i j} p\left(y_{i j}\right)=a$ by $\left(2.2^{\prime}\right)$. Hence, $V_{A}(V)=B$.

Let $\mathfrak{B}_{l}$ be the set of all intermediate rings $B^{\prime}$ of $A / B$ such that ${ }_{B^{\prime}} B_{B}^{\prime}<\oplus_{B^{\prime}} A_{B}$ and ${ }_{B^{\prime}} B^{\prime} \otimes_{B} A_{A} \rightarrow{ }_{B^{\prime}} A_{A}\left(b^{\prime} \otimes a \mapsto b^{\prime} a\right)$ splits, and $\mathfrak{B}_{\imath}$ the set of all intermediate rings $V^{\prime}$ of $V / C$ such that ${ }_{V^{\prime}} V^{\prime}<\oplus_{V^{\prime}} V$ and ${ }_{V^{\prime}} V^{\prime} \otimes_{C} V_{V} \rightarrow_{V^{\prime}} V_{V}$ $\left(v^{\prime} \otimes v \mapsto v^{\prime} v\right)$ splits. Similarly, we can consider the sets $\mathfrak{B}_{r}$ and $\mathfrak{Y}_{r}$. Finally, let $\mathfrak{B}$ be the set of all intermediate rings $B^{\prime}$ of $A / B$ such that $B^{\prime} / B$ is separable and ${ }_{B^{\prime}} B_{B^{\prime}}^{\prime}<\oplus_{B^{\prime}} A_{B^{\prime}}$, and $\mathfrak{B}$ the set of all intermediate rings $V^{\prime}$ of $V / C$ such that $V^{\prime} / C$ is separable. Needless to say, $\mathfrak{B}$ is a subset of $\mathfrak{B}_{l}$. (In [7], $\mathfrak{B}$ was denoted as $\mathfrak{D}$.)

(6) Let $B^{\prime}$ be an intermediate ring of $A / B$ with $V^{\prime}=V_{A}\left(B^{\prime}\right)$. If ${ }_{B^{\prime}} B_{B}^{\prime}<\oplus_{B^{\prime}} A_{B}$ or ${ }_{B} B_{B^{\prime}}^{\prime}<\oplus_{B} A_{B^{\prime}}$ then $V_{A}\left(V^{\prime}\right)=B^{\prime}$. Especially, if $B^{\prime}$ is in $\mathfrak{B}_{l}$ (resp. $\mathfrak{B}_{r}$ ) then $V^{\prime}$ is in $\mathfrak{B}_{l}$ (resp. $\mathfrak{B}_{r}$ ) and $V_{A}\left(V^{\prime}\right)=B^{\prime}$.

Proof. Let $p: A \rightarrow B^{\prime}$ be a $B^{\prime}$-B-epimorphism which induces the 
identity map on $B^{\prime}$. Then, by $\sum_{j} p\left(x_{i j}\right) y_{i j} \in V^{\prime}$ and $\left(2.1^{\prime}\right)$, we obtain for every $b^{\prime \prime} \in V_{A}\left(V^{\prime}\right), \quad p\left(b^{\prime \prime}\right)=\sum_{i, j} p\left(x_{i j}\right) y_{i j} b^{\prime \prime} v_{i}=b^{\prime \prime} \sum_{i, j} p\left(x_{i j}\right) y_{i j} v_{i}=b^{\prime \prime}$. This proves $V_{A}\left(V^{\prime}\right)=B^{\prime}$. Henceforth, we assume further that ${ }_{B^{\prime}} B^{\prime} \otimes_{B} A_{A^{\prime}} \rightarrow{ }_{B^{\prime}} A_{A}$ $\left(b^{\prime} \otimes a \mapsto b^{\prime} a\right)$ splits. Then,. $V^{\prime} V^{\prime}<\oplus_{V^{\prime}} V$ by (1). We define a $V^{\prime}-V$-homomorphism $V \rightarrow V^{\prime} \otimes_{c} V$ by $v \mapsto \sum_{i} \sum_{j} p\left(x_{i j}\right) v y_{i j} \otimes v_{i}=\sum_{i} \sum_{j} p\left(x_{i j}\right) y_{i j} \otimes v_{i} v$ (cf.. the definition of $\xi$ and $\left.\left(2.1^{\prime}\right)\right)$. Then, $\sum_{i, j} p\left(x_{i j}\right) v y_{i j} v_{i}=v$ means that ${ }_{{ }^{\prime}} V^{\prime} \otimes_{C} V_{V^{\prime}} \rightarrow_{\nabla^{\prime}} V_{V}\left(v^{\prime} \otimes v \mapsto v^{\prime} v\right)$ splits. Hence, $V^{\prime} \in \mathfrak{B}_{l}$.

(7) If $V^{\prime}$ is in $\mathfrak{B}_{\imath}$ (resp. $\mathfrak{B}_{r}$ ) then $B^{\prime}=V_{A}\left(V^{\prime}\right)$ is in $\mathfrak{B}_{\imath}$ (resp. $\mathfrak{B}_{r}$ ) and $V_{A}\left(B^{\prime}\right)=V^{\prime}$.

PRoof. Since ${ }_{V^{\prime}} V^{\prime} \otimes_{C} V_{V} \rightarrow{ }_{\nu} V_{V}\left(v^{\prime} \otimes v \mapsto v^{\prime} v\right)$ splits, there exists an element $\sum_{k} v_{k}^{\prime} \otimes u_{k} \in\left(V^{\prime} \otimes_{C} V\right)^{V^{\prime}}$ such that $\sum_{k} v_{k}^{\prime} u_{k}=1$. Obviously, $\sum_{k} v_{k}^{\prime} x_{i j} u_{k}$ $\in B^{\prime}$ and ${ }_{B^{\prime}} B_{B}^{\prime}<\oplus_{B^{\prime}} A_{B}$ by (1). Next, we consider an arbitrary left $V^{\prime}$-epimor phism $q: V \rightarrow V^{\prime}$ which induces the identity map on $V^{\prime}$, and define the map ८: $A \rightarrow B^{\prime} \otimes_{B} A$ by $a \mapsto \sum_{i, j} \sum_{k} v_{k}^{\prime} x_{i j} u_{k} \otimes y_{i j} q\left(v_{i}\right) a$. By $\left(2.1^{\prime}\right)$, we have $\sum_{i, j, k} v_{k}^{\prime} x_{i j} u_{k} v \dddot{y}_{i j} q\left(v_{i}\right) b^{\prime} a=\sum_{k} v_{k}^{\prime} q\left(u_{k} v\right) b^{\prime} a=b^{\prime} \sum_{i, j, k} v_{k}^{\prime} x_{i j} u_{k} v y_{i j} q\left(v_{i}\right) a\left(b^{\prime} \in B^{\prime}\right.$, $v \in V)$. Then, regarding $B^{\prime} \otimes_{B} A$ as a submodule of $A \otimes_{B} A$, we see that $\iota$ is a $B^{\prime}-A$-homomorphism and $\sum_{i, j, k} v_{k}^{\prime} x_{i j} u_{k} y_{i j} q\left(v_{i}\right) a=\sum_{k} v_{k}^{\prime} q\left(u_{k}\right) a=a$. Hence, $B^{\prime} \in \mathfrak{B}_{l}$. Moreover, if $v^{\prime \prime} \in V_{A}\left(B^{\prime}\right)$ then $v^{\prime \prime}=v^{\prime \prime} 1=$ $v^{\prime \prime} \sum_{i, j, k} v_{k}^{\prime} x_{i j} u_{k} y_{i j} q\left(v_{i}\right)=\sum_{k} v_{k}^{\prime} q\left(u_{k} v^{\prime \prime}\right) \in V^{\prime}$, which means $V_{A}\left(B^{\prime}\right)=V^{\prime}$.

Now, as a combination of (6) and (7), we readily obtain the main part of $[7,(1.3)]$ :

THEOREM 1. Let $A / B$ be an H-separable extension.

(a) $B^{\prime} \mapsto V_{A}\left(B^{\prime}\right)$ and $V^{\prime} \mapsto V_{A}\left(V^{\prime}\right)$ are mutually converse 1-1 correspondences between $\mathfrak{B}_{l}$ (resp. $\mathfrak{B}_{r}$ ) and $\mathfrak{B}_{l}$ (resp. $\mathfrak{B}_{r}$ ).

(b) $B^{\prime} \mapsto V_{A}\left(B^{\prime}\right)$ and $V^{\prime} \mapsto V_{A}\left(V^{\prime}\right)$ are mutually converse 1-1 correspondences between $\mathfrak{B}$ and $\mathfrak{B}$.

Proof. It remains only to prove (b). First, we claim $\mathfrak{B} \subset \mathfrak{B}_{l}$. Given $V^{\prime} \in \mathfrak{B}$, we put $C^{*}=V_{V^{\prime}}\left(V^{\prime}\right), \quad V^{\prime \prime}=V_{V}\left(V^{\prime}\right)$, and $U=V_{V}\left(C^{*}\right)$. Since $V^{\prime} / C$ is separable, we have ${ }_{V^{\prime \prime}} V^{\prime \prime}{ }_{V^{\prime \prime}}<\oplus_{V^{\prime \prime}} V_{V^{\prime \prime}}$ by (1). Recalling that $V_{C}$ is f.g. projective by (3), we see that $V^{\prime \prime}{ }_{C}$ is f.g. projective. Combining this with the separability of $C^{*} / C$ (cf. [1, Theorem 2.3]), one will readily see that $V^{\prime \prime} c^{*}$ is f.g. projective, so that $C^{*} c^{*}<\otimes V^{\prime \prime} c^{*}$. On the other hand, since $V^{\prime} / C^{*}$ is central separable by $\left[1\right.$, Theorem 2.3], we have $V^{\prime} \otimes_{c^{*}} V^{\prime \prime} \cong V^{\prime} \cdot V^{\prime \prime}=U$ by [1, Theorem 3. 1] and ${ }_{V} U_{V}<\oplus_{V} V_{D}$ by (1). Hence, ${ }_{V} V^{\prime}<\oplus V_{V^{\prime}}$ and $V^{\prime} \in \mathfrak{B}_{l}$. Moreover, if we set $B^{\prime}=V_{A}\left(V^{\prime}\right)$ then ${ }_{B^{\prime}} B_{B^{\prime}}^{\prime}<\oplus_{B^{\prime}} A_{B^{\prime}}$ by $(1)$ and ${ }_{B^{\prime}} B^{\prime} \otimes_{B^{\prime}} A_{A}$ $\rightarrow{ }_{B^{\prime}} A_{A}\left(b^{\prime} \otimes a \mapsto b^{\prime} a\right)$ splits by $(\mathrm{a})$. Hence $B^{\prime} \mid B$ is separable and $B^{\prime} \in \mathfrak{B}$. Similarly, we can prove that $V_{A}\left(B^{\prime}\right)$ is in $\mathfrak{B}$. Now, the rest of part of the proof is immediate by (a). 
Corollary. Let $A / B$ be an $H$-separable extension with $V_{A}(V)=B$, and $B^{\prime}$ is in $\mathfrak{B}$. If the center $Z^{\prime}$ of $B^{\prime}$ is contained in the center of $B$ then $V_{B^{\prime}}\left(V_{B^{\prime}}(B)\right)=B$ (and conversely).

Proof. By Theorem 1 (b), $V^{\prime}=V_{A}\left(B^{\prime}\right)$ is separable over $C$ and $V_{A}\left(V^{\prime}\right) \cap V^{\prime}=B^{\prime} \cap V^{\prime}=Z^{\prime}$. Hence, $V^{\prime}$ is a central separable algebra over $Z^{\prime}$ by [1, Theorem 2.3]. Since $Z^{\prime}$ is contained in $V_{B}(B$, ) we have $V=V^{\prime} \otimes_{Z \prime} V_{V}\left(V^{\prime}\right)=V^{\prime} \otimes_{Z^{\prime}} V_{B^{\prime}}(B)$ by [1, Theorem 3. 1]. It follows then $V_{B^{\prime}}\left(V_{B^{\prime}}(B)\right)=B^{\prime} \cap V_{A}\left(V_{B^{\prime}}(B)\right)=V_{A}\left(V^{\prime}\right) \cap V_{A}\left(V_{B^{\prime}}(B)\right)=V_{A}(V)=B$.

(8) Every ring-homomorphism $\sigma$ of $A$ leaving every element of $B$ invariant is a monomorphism.

Proof. In fact, if $\sigma(a)=0$ then $0=\sum_{i, j} x_{i j} \sigma\left(y_{i j}\right) \sigma(a) \sigma\left(v_{i}\right)=a$ by (2.1).

We shall conclude this note with the following theorem.

THEOREM 2. Let $A / B$ be an $H$-separable extension, and $\sigma$ a ringendomorphism of $A$ which leaves every element of $B$ invariant.

(a) If $V_{A}(\sigma(A))=C$ then $\sigma$ is an automorphism.

(b) If $\sigma$ leaves every element of $C$ invariant, then $\sigma$ is an automorphism. Especially, if $C \subset B$ then $\sigma$ is an automorphism.

(c) If ${ }_{B} B<\oplus_{B} A$ or $B_{B}<\oplus A_{B}$ then $\sigma$ is an automorphism.

Proof. Let $A^{\prime}=\sigma(A)$, and $C^{\prime}=V_{A}\left(A^{\prime}\right)$.

(a) To be easily seen, $\sum_{s, j} \sigma\left(x_{r s}\right) y_{r s} x_{i j} \sigma\left(y_{i j}\right)$ is in $C^{\prime}=C$. Hence, if $a$ is an arbitrary element of $A$ then by $\left(2.1^{\prime}\right)$ we have $\sigma\left(\sum_{i, j} x_{i j} \sigma\left(y_{i j}\right) a \sigma\left(v_{t}\right)\right)$ $=\sum_{r, s} \sigma\left(x_{r s}\right) y_{r s}\left(\sum_{i, j} x_{i j} \sigma\left(y_{i j}\right) a \sigma\left(v_{i}\right)\right) v_{r}=\sum_{r, i}\left(\sum_{s, j} \sigma\left(x_{r s}\right) y_{r s} x_{i j} \sigma\left(y_{i j}\right)\right) a \sigma\left(v_{i}\right) v_{r}$ $=a \sum_{r, i} \sum_{s, j} \sigma\left(x_{r s}\right) y_{r s} x_{i j} \sigma\left(y_{i j}\right) \sigma\left(v_{i}\right) v_{r}=a \sum_{r, s} \sigma\left(x_{r s}\right) y_{r s} \sum_{i, j} x_{i j} \sigma\left(y_{i j} v_{i}\right) v_{r}=$ $a \sum_{r, s} \sigma\left(x_{r s}\right) y_{r s} v_{r}=a$. This together with (8) implies that $\sigma$ is an automorphism.

(b) Obviously, $A^{\prime}$ is an $H$-separable extension of $B$, and hence a separable extension of $B$ by (4). By (a), our proof will be complete if we can prove that $C^{\prime}$ coincides with $C$. We consider here the $C$-homomorphisms $f: C^{\prime} \otimes_{c} V \rightarrow V$ defined by $c^{\prime} \otimes v \mapsto c^{\prime} \sigma(v)$ and $g: V \rightarrow C^{\prime} \otimes_{c} V$ defined by $v \mapsto$ $\sum_{i} \sum_{j} \sigma\left(x_{i j}\right) v \sigma\left(y_{i j}\right) \otimes v_{i}$. Then, by (2.3) $f g(v)=\sum_{i, j} \sigma\left(x_{i j}\right) v \sigma\left(y_{i j} v_{i}\right)=v$. On the other hand, $g f\left(c^{\prime} \otimes v\right)=\sum_{i} \sum_{j} \sigma\left(x_{i j}\right) c^{\prime} \sigma(v) \sigma\left(y_{i j}\right) \otimes v_{i}=c^{\prime} \sum_{i} \sigma\left(\sum_{j} x_{i j} v y_{i j}\right)$ $\otimes v_{i}=c^{\prime} \sum_{i, j} x_{i j} v y_{i j} \otimes v_{i}=c^{\prime} \otimes v$. Hence, $C^{\prime} \otimes_{c} V \cong V$. Since, $V_{c}$ is f.g. projective by $(3)$ and ${ }_{C^{\prime}} C_{C^{\prime}}^{\prime}<\oplus_{C^{\prime}} V_{C^{\prime}}$ by $(1), C_{C}^{\prime}$ is an f.g. projective module of rank 1. Hence, by [2, Corollaire du Théorème 1], it follows $C^{\prime}=C$.

(c) This is immediate by (5) and (b). 


\section{References}

[1] M. Auslander and O. Goldman: The Brauer group of a commutative ring, Trans. Amer. Math. Soc., 97 (1960), 367-409.

[2] N. Bourbaki: Élements de Mathématique, Algèbre Commutative, Chaps. 1-2, Actualités Sci. Ind. 1290, Hermann, Paris, 1961.

[ 3 ] K. Hirata : Some types of separable extensions of rings, Nagoya Math. J., 33 (1968), 107-115.

[4] T. Nakamoto: On $Q F$-extensions in an $H$-separable extension, Proc. Japan Acad., 50 (1974), 440-443.

[5] K. Sugano: Note on semi-simple extensions and separable extensions, Osaka J. Math., 4 (1968), 265-270.

[6] K. Sugano: On centralizer in separable extensions II, Osaka J, Math., 8 (1971), 465-469.

[7] K. Sugano: On some commutor theorems of rings, Hokkaido Math. J., 1 (1972), 242-249.

(Received October 21, 1974) 